\title{
Antimycotic screening of 58 Malaysian plants against plant pathogens
}

\begin{abstract}
Ethanolic extracts of 58 Malaysian plants belonging to 24 different families were screened for antifungal activity against seven plant pathogens using the filter paper disc diffusion technique. Two varieties of Piper betle, showed strong activity against all the pathogens tested (Colletotrichum capsici, Fusarium pallidoroseum, Botryodiplodia theobromae, Alternaria alternata, Penicillium citrinum, Phomopsis caricae-papayae and Aspergillus niger), with inhibition diameters significantly $(\mathrm{P}<0 \cdot 01)$ bigger than $2.5 \mathrm{mg}$ mli 1 prochloraz or 10 mg mlī 1 clotrimazole. The minimum inhibitory concentrations of the ethanolic extracts of $\mathrm{P}$. betle against these plant pathogens ranged between $0.01 \mathrm{mg} \mathrm{mlī} 1$ and $1 \mathrm{mg}$ mlī 1 . Thirtyfour other plants (Kucing gala, Limau batik, Bertholletia excelsa, Bixa orellana, Caesalpinia pulcherrima, Cerbera odollam (fruits and leaves), Colocasia gigantea, Curcuma domestica, Curcuma manga, Derris eliptica, Elephantopus scaber, Eleusine indica, Eugenia polyantha, Euphorbia hirta, Euphorbia tirucalli, Gardenia florida, Hedyotis auricularia, Hibiscus rosasinensis, Juniperus chinensis (three varieties), Lawsonia inermis, Lecythis ollaria, Mentha arvensis, Mimusops elengi, Ocimum sanctum, Phyllanthus niruri, Piper nigrum, Piperomia pellucida, Pedilanthus tithymaloides, Polygonum minus, Spondias dulcis, Solanum nigrum, Tinospora tuberculata) showed selective antifungal activity, while 21 species were inactive.
\end{abstract}

Keyword: Antifungal; Piper betle; Plant pathogens; Malaysian plants 